\title{
INTERACTION IN A SENSITIVE HOUSE
}

Future room that senses human mood and respond to it

Henry Thomas

France Telecom R\&D, 4 rue du Clos Courtel, BP 91226, 35512 Cesson Sévigné cedex, France-mail:henry.thomas@francetelecom.com

\begin{abstract}
We describe a sensitive and multimedia house conceived at France telecom R\&D Studio creative, and the way we experiment interface for that house. Interaction can be explicit, such as bike, carpet-pad that control directly multimedia content. Interaction can also be indirect, making use of emotion analysis. We are working with multimedia artist Naoko Tosa to investigate the latter category of interactions.
\end{abstract}

Key words: interaction, emotions, pervasive computing

\section{INTRODUCTION}

The Creative studio is a part of France Telecom's R\&D centre. It was launched in 1997 to promote innovation in communication services. Its main thrust is to build up usages, services and customer awareness in an innovative process traditionally governed by a technical and scientific structure. The Creative studio complement the R\&D approach with new methods borrowed from human sciences, creativity and artistic creation. Coming up with ideas gathered from different input, we design conceptservices, simple illustrations together with scenarios of use. From the scenarios and illustration, we obtain reactions, comments from marketing teams, projects teams or future customers, or from potential users. This helps us to refine the concept, to end up with a precise definition of the product. 


\section{THE SENSITIVE HOUSE}

We are testing our services in a simulation of a house, which is around 100 square meters. It is designed to be modular and to allow a rapid reconfiguration, either from the technical side (electronic, routing of video, sound and computer signals) or from the furniture point of view. Thus, we are able to adapt to different ways of life. The aim is to have a familiar environment, in which user can describe their existing practice, and also develop new ones. We use the house in several different ways, which can be contradictory sometimes:

- A domestic house to test automated element of comfort.

- A secure house in which we can test the security of persons and goods.

- An adaptative house in which we develop new ideas and concept about the living space and its components (ambient intelligence, pervasive computing).

- A sensitive and multimedia house which is more oriented on leisure, permanently connected to the world. It relies on the perspective of broadband access, and on the ability for the user to interact naturally and easily with services. It is this latter house that is the subject of this article.

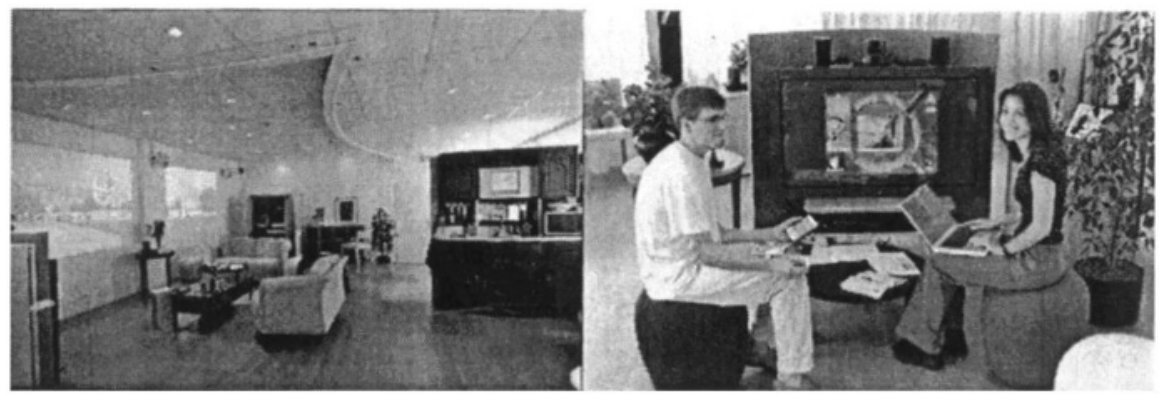

Figure 1. The sensitive house. Left side, the view shows from left to right 1) the back a plasma display (see right picture), two projection screen to create data projection, or panorama live videos, 3 ) the kitchen space, which integrate a display screen. The right picture displays the large plasma screen, also use for video conferencing, with to $\mathrm{PhD}$ students mimicking end-users.

To interact with the user, we devised information appliances [3] in the shape of objects. An information appliance is an intelligent object that performs one function (for instance give the weather forecast with a single push on an unique button). The different appliances in the house cooperate in order to provide the desired result. In the sensitive house, you will find a variety of sensors and object that allows direct control over "things" 
(comfort, medias) and other sensors that work in a more elaborate and indirect way, such as emotions.

\section{HETEROGENEITY OF SENSOR}

Our goal is to provide different interactors in the house, suited for each member of the family, depending on his needs and moods. We developed, alone or in cooperation with artists and designers, several concept services.

- A bicycle, that enable users to navigate trough 3D content[2] and inside a travelogue[1].

- A sensitive carpet, similar in principle to a Playstation-2 dance pad, though this carpet is much wider : 2 meters by 3 meter, with an accuracy of $15 \mathrm{~cm}$. With the carpet, one can navigate in a 3D world.

- A video tracking system: a camera mounted on the ceiling is able to identify a point of light. The pictures and sounds of the room will be modified according to the movements.

All these navigation system were developed separately and do not interoperate. What if Jane user is tired does not want to bike, but want to sit on the floor and still interact? What if Jon wants to move freely inside the house? Jane could use the sensitive carpet, and Jon exploits the video tracking feature. We are reengineering our demos, so that input devices are all permutable, just like a PC gamer could use a Joystick, a game pad or a keyboard. To achieve this result, we choose to use the VRPN abstraction layer. VRPN met all of our requirements. "The Virtual-Reality Peripheral Network (VRPN) is a set of classes within a library and a set of servers that are designed to implement a network-transparent interface between application programs and the set of physical devices (tracker, etc.) used in a virtual-reality (VR) system. The idea is to have a PC or other host at each $V R$ station that controls the peripherals (tracker, button device, haptic device, analog inputs, sound, etc)" [4]. VRPN application domain was only the world of 3D modeling and cinema studio. We propose to use it in the house. Using this layer will open combinations that were not suspected before: navigation in a CDROM with a bike or a carpet, 3D world navigation via a carpet. Apart from the multi sensors aspect, this opens up possibilities for disabled people. This allows to simply switching to a "preferred input device". 


\section{INTUITIVE CONTROL}

However, all these systems are used for explicit control / movement into $2 \mathrm{D}$ or 3D world, or to control a video playback. We want to develop systems that allow intuitive control. The aim of such systems is to be able to address issues such as:

- How Jane can set the ambient of the house without having to use a control interface that explicitly addresses all lights and blinds?

- How John can browse a huge CD collection (either local or network based), looking for a music that suits its mood?

One answer is to detect the user mood and to (re)act accordingly. The reaction could be to propose an ambient for the house (combination of light sound, pictures). The same way, we could find a record in a huge library of CD that is adequate for John's mood.

We established a partnership with the Japanese multimedia artist Naoko Tosa to integrate her interactive piece of work "The Zenetic computer" [5] inside the sensible house. ZENetic Computer is an interactive experience that evokes "self-awakening, a particular cognitive response to processing reality via subliminal consciousness. It uses stories portrayed in sumi-e (ink painting), haiku and kimono which display features of eastern philosophy, and Zen in particular. Visitors create their own sumi-e and stories on a large rice paper screen, while learning about Zen, Japanese art, and themselves.

The ZENetic computer uses the ink painting and interactions to induce an emotional state for the visitor, and then tries to bring the visitor, through a sequence of interactions to a higher awakening. We believe that such devices can be used in the future to interact with services. Imagine a scenario in which you come back home. You do not want to interact with a PC computer to specify an ambient (music, videos etc.). You could just sit and place different special objects on a surface, or do a drawing, just like the one we often do during a boring phone call. The house can then analyze the pattern of object, or the drawing to induce your mental state, and go through patterns learnt to find the ambient that fits your mood, without having to go through introspection. 


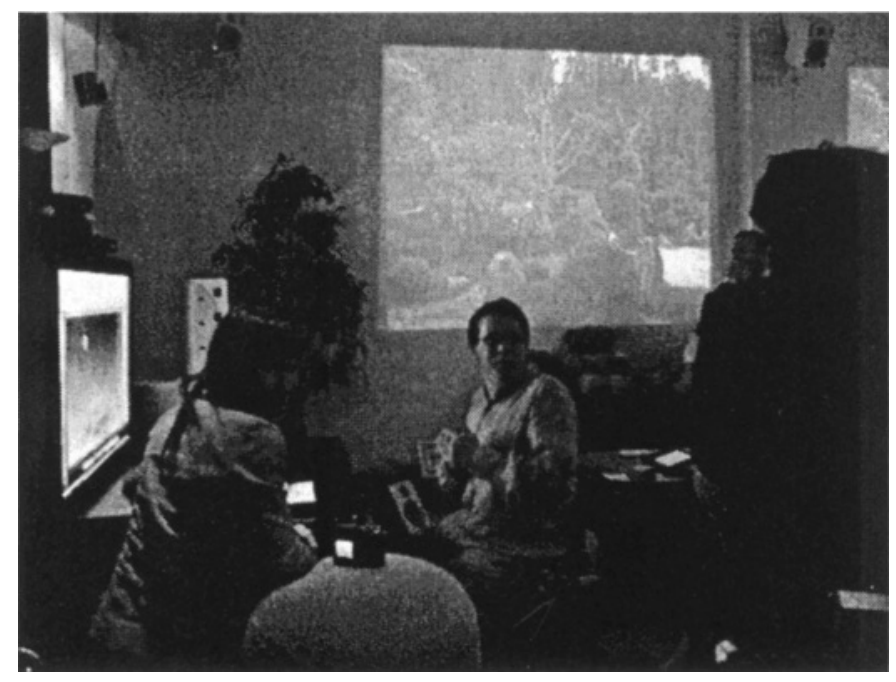

Figure 2. The Zenetic computer in the sensitive house. The plasma screen is used for main display, while a large rock garden (in front of the author) integrates a sensitive screen for interaction. Smells are emitted by the yellow box in the back, and videos are projected on the wall behind. A very dark ambient is used.

\section{CONCLUSION}

The field of domestic interfaces and interaction is quite wide and we focus mainly on the two directions described:

- Low level interactors such a bike, carpet that we use to directly control media (video, pictures, 3D)

- High level interactors that deal with emotion, and allow the user to explore a non explicit dialog with the house, based on the building of a language, specific the house and its inhabitants.

\section{REFERENCES}

[1] "IBIS interactive travelogue", Rob White, 2000, http://www.veloquence.com

[2] "Using a Bicycle Interface for Interaction and Games", Henry Thomas, Rob White,. 10th International Conference on Human - Computer Interaction, June 22-26, 2003

[4] VRPN, University of northern California http://www.cs.unc.edu/Research/vrpn/

[3] “The Invisible computer", Donald A. Norman, MIT Press, 1999

[5] Naoko Tosa, Chapter 19: Expression of emotion unconsciousness with art and technology, "Affective minds", Elsevier, pp. 183201 (200) 